\section{$\underset{\substack{\text { hommes } \\ \text { \& migrations }}}{ }$}

\section{Hommes \& migrations}

Revue française de référence sur les dynamiques

migratoires

$1302 \mid 2013$

Le Japon, pays d'immigration?

\title{
Les travailleur(se)s domestiques migrant(e)s en Asie
}

\section{Ruri Ito}

\section{(apenEdition \\ Journals}

\section{Édition électronique}

URL : http://journals.openedition.org/hommesmigrations/2476

DOI : 10.4000/hommesmigrations.2476

ISSN : 2262-3353

\section{Éditeur}

Musée national de l'histoire de l'immigration

Édition imprimée

Date de publication : 1 avril 2013

Pagination : 33-39

ISBN : 978-2-919040-22-3

ISSN : $1142-852 X$

\section{Référence électronique}

Ruri Ito, "Les travailleur(se)s domestiques migrant(e)s en Asie », Hommes \& migrations [En ligne], 1302 | 2013, mis en ligne le 31 décembre 2015, consulté le 20 avril 2019. URL : http://

journals.openedition.org/hommesmigrations/2476; DOI : 10.4000/hommesmigrations.2476 


\title{
LES TRAVAILLEUR(SE)S DOMESTIQUES MIGRANT(E)S EN ASIE
}

par RURI ITO, professeure de sociologie, université Hitotsubashi ${ }^{1}$

\author{
En Asie du Sud-Est, de plus en plus de femmes migrent pour \\ occuper des emplois domestiques. L'internationalisation \\ des métiers du care et de la reproduction d'une division sexuée \\ du travail explique le développement de cette migration \\ féminine, notamment à Hong Kong et à Singapour. \\ Au Japon, le poids des discriminations de genre tend plutôt \\ à valoriser le modèle de la femme au foyer. Les métiers \\ domestiques souffrent dans ce pays d'un vide juridique, \\ malgré l'adoption récente d'une convention par l'Organisation \\ internationale du travail.
}

\section{Comprendre la position "périphérique" du Japon dans la division internationale du travail reproductif}

Faire le ménage, la lessive, la cuisine, les courses, s'occuper des enfants, les accompagner à l'école, ou bien prendre en charge les personnes dépendantes, tout cela fait partie des obligations de notre vie quotidienne. La répétition quotidienne de ces tâches (désignées ci-dessous par "travail reproductif") est dans de nombreuses sociétés assumée par les femmes au nom de leur rôle d'épouse, de mère, de grand-mère, de belle-fille ou de fille au sein de la famille. L'expression "division internationale du travail reproductif" (DITR) désigne le phénomène par lequel des migrants, essentiellement des femmes, passent les frontières pour travailler dans l'espace privé qu'est le "foyer" et pour accomplir les tâches domestiques que nous venons d'évoquer. Depuis le milieu des années 1970, du fait de la croissance rapide des "économies nouvellement industrialisées (NIEs)" telles que Singapour et Hong Kong ou les pays du Golfe qui s'enrichissaient grâce à la flambée des prix du pétrole brut, 
"le marché des travailleurs domestiques" a subitement pris de l'importance dans cette partie du monde, marquant le début d'un phénomène qu'on nommera la "féminisation de la migration internationale".

Curieusement, ce phénomène n'a pas pris la même forme au Japon. Il s'est traduit essentiellement par l'arrivée massive de femmes d'Asie du Sud-Est dans l'industrie de la vie nocturne (entertainment) - couplée avec l'augmentation des mariages entre ces femmes et les hommes japonais ${ }^{2}$-, et non par le développement du travail
"Le marché des

travailleurs domestiques"

a subitement pris

de l'importance dans cette

partie du monde, marquant

le début d'un phénomène

qu'on nommera la

"féminisation de la migration internationale". reproductif. Sur ce plan, le Japon a occupé et continue à occuper une position périphérique. "Périphérique" dans deux sens.

D'abord, pour le gouvernement japonais, l'introduction de travailleurs migrants reste l'option à éviter pour maintenir l'"harmonie sociale", encore plus s'il s'agit de femmes, donc si cela concerne l'ordre du genre, infrastructure du nationalisme. Ensuite, vu du côté des travailleur(se)s domestiques migrant(e)s ${ }^{3}$, le Japon comme pays de destination est une option relativement moins attrayante que d'autres possibilités telles que le Canada, les États-Unis, Hong Kong ou Singapour, ne serait-ce que du point de vue de la langue.

Il s'agit donc de suivre le développement du travail reproductif en Asie, en l'occurrence à Hong kong et à Singapour, d'observer, d'une part, ses particularités sur le plan de la politique d'État et, d'autre part, d'éclairer la particularité du cas japonais par rapport aux autres pays d'Asie.

\section{Le renforcement du travail reproductif des classes moyennes}

C'est en 1995, lors de la préparation de la $4^{e}$ Conférence mondiale des femmes à Beijing, que diverses ONG ont commencé à tirer la sonnette d'alarme sur l'apparition de nouvelles formes d'esclavage avec l'émergence du marché international du travail domestique, déplaçant massivement des femmes pauvres de divers pays d'Asie. Selon ces ONG, en 1994, le nombre de migrantes exerçant l'activité de travailleur domestique en Asie se situait entre 1 et 1,7 million de personnes ${ }^{4}$. Ce phénomène n'a depuis cessé de s'accélérer. Actuellement, il est avéré que, pour le seul cas de l'Indonésie, environ 6 millions de femmes sont parties vers 42 pays $^{5}$. Quant aux Philippines, pour la seule année 2011, plus de 140000 personnes ont été officiellement embauchées à l'étranger dans le secteur du travail domestique ${ }^{6}$.

Des études sur le cas singapourien révèlent qu'avant même cette "féminisation de la migration internationale”, il existait déjà un régime colonial du transfert du travail reproductif. C'est le cas des femmes originaires du sud de la Chine, appelées "amahs", et dont la migration vers la ville de Singapour date du début du XXe siècle.

Traditionnellement, c'étaient les Cantonaises qui accomplissaient le travail reproductif pour les familles aisées - souvent européennes - dans la péninsule malaise ${ }^{7}$.

Cependant, le vieillissement des amahs ainsi que l'essor du besoin de main-d'œuvre féminine dans les usines, moteur de l'industrialisation orientée

\footnotetext{
2. Voir l'encadré sur les mariages mixtes dans ce même numéro d'Hommes \& Migrations (note des coordinateurs). 3. En français, le terme "travailleur(se)s domestiques" pourrait être interprété avec une connotation négative, pour des raisons historiques. Nous avons opté ici pour cette expression afin de nous conformer à la version française de la "Convention sur le travail décent des travailleurs et travailleuses domestiques" du Bureau international du travail, dont nous discuterons plus loin.4. Noeleen Heyzer, et al., The Trade in Domestic Workers: Causes, Mechanisms and Consequences of International Migration, Kuala Lumpur, Asian and Pacific Development Centre, 1995, p. 40. 5. http://www.idwn.info/news/domestic-workers- around-world-support-jalaprt-indonesia [Date de consultation: le 20 juin 2012] 6. Philippine Overseas Employment Administration (POEA), 2007-2011. http://www.poea.gov.ph/stats/2011Stats.pdf [Date de consultation : le 2 mai 2013] 7. Brenda S. A. Yeoh and Shirlena Huang, "Negotiating public space : strategies and styles of migrant female domestic workers in Singapore", in Urban Studies, vol. 35, n³, 1998, pp. 583-602. Pour un recueil de mémoires sur les "amahs", voir également, K. Gaw, Superior Servants: The Legendary Cantonese Amah of the Far East, Singapore, Oxford University Press, 1988.
} 
vers l'exportation des années 1960, ont conduit à l'épuisement graduel de ce bassin de main-d'œuvre domestique.

Vu le manque de services sociaux publics tels que la garde d'enfants, le gouvernement singapourien recrute en 1974 des travailleur(se)s domestiques migrants venu(e)s des Philippines et, ultérieurement, du Sri Lanka ou d'Indonésie, afin de pallier la pénurie de main-d'œuvre dans le travail reproductif et, surtout, de permettre aux femmes/mères locales diplômées de travailler. Sans entrer dans le détail, on peut dire que ce contexte historique s'applique globalement aussi à Hong Kong, un autre employeur majeur de travailleurs domestiques migrants.

Les gouvernements de Hong Kong et Singapour ont défini de façon rigoureuse les conditions dans lesquelles les migrantes pouvaient être embauchées. Ces gouvernements limitent le droit d'embaucher des travailleurs domestiques migrants aux couches aisées. Des règles ont été définies pour l'emploi d'un(e) travailleur(se) domestique migrant(e), telles que le revenu minimum, un espace minimal pour pouvoir le loger chez soi, l'obligation de payer une taxe et les frais de retour du travailleur domestique migrant, etc. Comme l'ont analysé Vivienne Wee et Amy Sim pour le cas de Hong Kong, cette politique est marquée par une dimension de classe $^{8}$. Le nombre de travailleurs domestiques migrants a augmenté de façon constante depuis, pour atteindre à Hong Kong à peu près 300000 aujourd'hui ${ }^{9}$. Cette politique reflète la volonté du gouvernement de renforcer l'infrastructure du travail reproductif des classes moyennes, afin de permettre aux femmes/ mères diplômées de contribuer à restructurer l'économie des années 1980-1990 et à augmenter sa compétitivité internationale.

\section{De l'encadrement des migrants à l'industrie de la migration}

Le séjour des travailleurs domestiques migrants est strictement limité dans le temps et les contrats d'embauche renouvelés tous les deux ou trois ans. Ces femmes n'ont pas la possibilité de faire venir des membres de leur famille. La possibilité de changer d'employeur en cours de contrat et de rester dans le pays après la fin d'un contrat est considé-

\section{Figure 1 : Évolution des personnels des services pour le soutien à la vie familiale au Japon (1975-2005)}

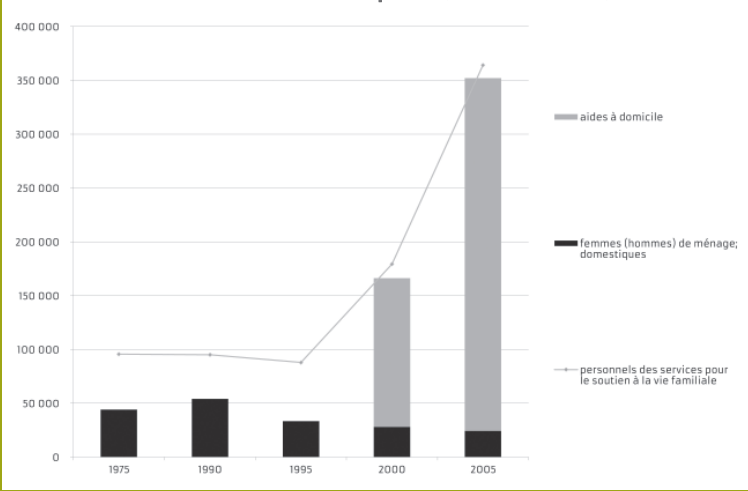

Sources : Recensement 1975 -2005, ministère des Affaires intérieures et de la Communication.

rablement limitée. La nature extrêmement stricte de ces règlementations s'est vue confirmée dans la décision récente de la Cour suprême de Hong Kong, qui a refusé à une employée de maison de nationalité philippine le droit de résidence permanente, bien qu'elle ait travaillé et vécu dans cette ville pendant presque vingt-sept ans ${ }^{10}$. 


\section{Tableau 1 : Situation des travailleurs domestiques au regard des lois nationales des divers pays d'Asie}

\begin{tabular}{r|c|c|c|c}
\hline & $\begin{array}{c}\text { Pris en compte } \\
\text { par la législation } \\
\text { nationale } \\
\text { sur le travail }\end{array}$ & $\begin{array}{c}\text { Droits fondamentaux } \\
\text { garantis par la } \\
\text { législation nationale } \\
\text { sur le travail }\end{array}$ & $\begin{array}{c}\text { Droits des travailleurs } \\
\text { domestiques migrants } \\
\text { équivalents à ceux } \\
\text { des autres travailleurs }\end{array}$ & $\begin{array}{c}\text { Droit de former } \\
\text { un syndicat } \\
\text { en vertu du droit } \\
\text { national } \\
\text { (droit syndical) }\end{array}$ \\
\hline Hong Kong & Oui & Oui & Oui & Oui \\
\hline Indonésie & Non & Non & Non & Oui \\
\hline Malaisie & Non & Non & Non & Oui \\
\hline Taïwan & En partie & Non & Oui & Non \\
\hline
\end{tabular}

Sources: "National laws and trade union barriers

to collective representation", in APWLD, The Right to Unite:

A Handbook for Domestic Worker Rights Across Asia, Changmai, 2010, p. 21. [http://www.apwld.org/pdf/Advance\%20

Domestic\%2oWorkers\%2oRights\%2065-85.pdf]

Les pays qui envoient les migrants contrôlent également les migrations, comme l'illustre l'exemple de l'Agence des Philippines pour l'emploi outre-mer. La multiplication des politiques concernant les travailleurs domestiques migrants a conduit à l'émergence d'une gigantesque industrie de l'émigration, composée d'agents de recrutement, de formation, de placement, de voyage, d'envoi d'argent, etc., liant les sociétés "importatrices" et "exportatrices".

Quelquefois, ces agences sont dirigées par d'anciennes travailleuses domestiques migrantes ellesmêmes, dont la trajectoire dépasse souvent la région d'Asie, ce qui leur permet de devenir de véritables entrepreneuses dans ce nouvel "espace social transnational11" constitué par le travail reproductif.

\section{La position "périphérique" du cas japonais}

La situation du Japon est-elle différente par rapport à celle de Hong Kong ou de Singapour ? Dans ce pays, la révolution industrielle mobilisant la main-d'œuvre féminine, surtout des jeunes femmes, s'est produite au début du XXe siècle, bien plus tôt qu'à Singapour ou à Hong Kong. Le Japon est une économie "anciennement" industrialisée. Être employée de maison ou ouvrière d'usine, tel était le choix proposé aux jeunes femmes dans la période d'avant-guerre. En 1936, ont comptait 937000 travailleuses domestiques. Après-guerre, avec la diminution de la taille des ménages et la diversification des emplois pour les jeunes femmes, 
le nombre des travailleuses domestiques, après avoir atteint 300000 en 1955, commence à diminuer dans les années 1960 et ne compte plus que 50000 personnes en 1975, soit une diminution de plus de $80 \%$ en l'espace de vingt ans, avant d'atteindre environ 28000 en $2000^{12}$. Ce tarissement de la main-d'œuvre domestique n'a pas été compensé par l'arrivée des femmes migrantes. Tout d'abord, avec le Plan fondamental de la politique du travail de 1967, le gouvernement japonais annonce formellement la fermeture du marché du travail aux étrangers, à l'exception des métiers qualifiés de non remplaçables par les Japonais.

Au lieu de faire le choix du recours à la main-d'œuvre étrangère, c'est le renforcement de la division sexuée du travail fondée sur le modèle "l'homme au travail / la femme au foyer" - une nouvelle version de l'idéologie "ryôsai kenbo (bonne épouse, mère sage $)^{13}$ " inventée au moment de la modernisation de l'ère de Meiji -, qui s'installe et se traduit dans les pratiques de l'emploi. Les entreprises n'embauchent les femmes que jusqu'à ce qu'elles se marient ou les obligent à quitter leur emploi à la naissance de leur premier enfant et n'acceptent pas leur réintégration une fois que les enfants ont grandi. Ces pratiques étaient si répandues dans la société nommée "kigyô-chûshin shakai (société centrée sur les entreprises) ${ }^{14}$ " qu'elles étaient difficiles à contrer, tant elles étaient considérées comme faisant partie du "bon sens". Elles étaient justifiées par la croissance économique spectaculaire d'une part $\mathrm{t}^{15}$ et, d'autre part, par le nivellement du niveau de vie ("le Japon, une vaste classe moyenne"), produisant l'image d'une société faussement "égalitaire ${ }^{16 " . ~}$ À partir des années 1960, non seulement les travailleuses domestiques sont moins nombreuses, mais ce métier devient essentiellement celui des femmes d'un âge moyen et avancé. Les travailleuses domestiques n'acceptaient plus d'être logées chez l'employeur. Ce sont les agences mandataires et prestataires - à peu près 1200 agences en 199017 qui font le lien entre les employeurs particuliers et les travailleuses domestiques dont le nombre ne cesse de diminuer.

\section{Le primat de la division sexuée des tâches}

La politique d'immigration du gouvernement japonais est donc diamétralement opposée à celle de Singapour ou de Hong Kong. Sur le genre, sa politique est contradictoire. Alors que le Japon mettait en œuvre une série de réformes pour ratifier la Convention pour l'élimination de toutes formes de discriminations contre les femmes (ratifiée en 1985), il a introduit au même moment deux mesures qui favorisent le modèle de la "femme au foyer" : l'une portant sur les pensions de retraite (1986), l'autre sur l'exonération d'impôt sur le revenu des époux (1987). La première mesure a créé une catégorie de personnes (catégorie 3) qui ne cotisent pas pour l'assurance retraite, mais sont couvertes par les cotisations de leur conjoint. La seconde mesure Les entreprises n'embauchent les femmes que jusqu'à ce qu'elles se marient ou les obligent à quitter leur emploi à la naissance de leur premier enfant et n'acceptent pas leur réintégration une fois que les enfants ont grandi. favoriseles couples à double revenu, à condition que le revenu de l'un(e) - souvent celui de la femme - reste inférieur à un seuil fixe (à peu près 1 million de yens par an) en l'exonérant

12. Kazuko Koizumi (dir.), Jochû ga ita Shôwa (Les Bonnes de l'ère Shôwa), Kawade shobô shinsha, 2012

13. Ce slogan fut couplé avec son versant masculin, "fukoku kyôhei (pays riche, armée forte)". Shizuko Koyama, Ryôsai Kenbo to iu kihan (La Norme de bonne épouse, mère sage), Keisô shobô, 1991. Tomoko Nakamatsu, "Unpaid domestic work : gender, State policy and the labour market in Japan", in Shirlena Huang, Brenda S. A. Yeoh, Noor Abdul Rahman (dir.), art. cit., pp. 364-379.

14. Mari Osawa, Kigyô-chûshin shakai wo koete: Gendai Nihon shakai wo "gendâ" de yomu (Au-delà de la société centrée sur les entreprises: Lire la société japonaise avec le concept du genre), Jiji Tsushin, 1993.

15. Mary Brinton, Women and the Economic Miracle: Gender and Work in Postwar Japan, University of California Press, 1994.

16. Cette image d'une société égalitaire s'est répandue avec la publication du rapport de l'OCDE de 1976. Malcolm C. Sawyer, “Income distribution in OECD countries", OECD Economic Outlook, Occasional Studies, Paris, OECD, 1976

17. Eiko Shinotsuka, Ai Yokomura, "Kôreika Jidai no Joshi Rôdô : Tsukisoifu no rekishiteki hensen to genjyô" ("Le travail féminin à l'ère

du vieillissement de la population : l'évolution historique et la situation actuelle des 'femmes d'accompagnement"),

in Shakai Hoshô Kenkyû, vol. 26, n 3, 1990, p. 271. 
d'impôt ${ }^{18}$. En plus de maintenir le modèle dominant de la division sexuée du travail, ces deux mesures favorisent les milieux aisés, qui peuvent se permettre de vivre sur un seul revenu ${ }^{19}$. Alors qu'à Singapour ou à Hong Kong ces mesures privilégiant les couches aisées sont mises en œuvre dans le but de mobiliser les femmes/mères diplômées sur le marché du travail, au Japon elles ont l'effet inverse.

En ce qui concerne l'emploi des travailleurs domestiques migrants, le gouvernement japonais ne l'autorise que pour les foyers Alors qu'à Singapour d'étrangers(foyersàhauts reveou à Hong Kong ces mesures privilégiant les milieux aisés sont mises en œuvre

dans le but de mobiliser les femmes/mères diplômées sur le marché du travail, au Japon elles ont l'effet inverse. de la diplomatie, entre autres) Le nombre d'employés étrangers à domicile qui travaillent chez des résidents étrangers est estiméà environ 3000 (leur statut de résidence est celui domestiques migrants. Un des rares changements est l'introduction de l'assurance des soins de longue durée en $2000^{20}$. Sous la pression du vieillissement accéléré de la population, cette assurance a externalisé le "care” pour les personnes âgées dépendantes, jusque-là fourni dans le cadre de la famille. Elle a créé une nouvelle catégorie de métier, les "home helpers" ("aides à domicile"). Ces home helpers, pour la plupart des femmes, sont estimés à 327000 en 2005 (fig. 1) ${ }^{21}$. Il faut remarquer que pour les femmes migrantes qui se sont installées au Japon après s'être mariées, ce métier est à présent une des rares options d'emploi qui leur permette de s'intégrer dans le marché du travaile 22 . d'“activité spécifique”), ce qui, en comparaison des 300000 travailleurs domestiques présents à Hong Kong et des 209000 à Singapour, est très peu.

À la suite des récentes discussions pour encourager la venue au Japon d'“étrangers hautement qualifiés", le gouvernement a autorisé, à partir de mai 2012, cette nouvelle catégorie d'étrangers à être accompagnés de leur "employé de maison (kaji shiyônin)". Mais, fondamentalement, cette mesure ne change rien, car les Japonais n'ont toujours pas la possibilité d'embaucher des travailleurs

\section{La Convention des travailleurs domestiques de I'OIT}

Après avoir comparé les différentes modalités de mobilisation de la main-d'œuvre féminine par l'État et leurs conséquences sur le plan du travail reproductif, revenons sur la signification de l'adoption de la Convention sur le travail décent des travailleurs et travailleuses domestiques (Convention $n^{\circ}$ 189) en juin 2011 par l'Organisation internationale du travail ${ }^{23}$. Cette convention définit le travail domestique comme "le travail effectué au sein de ou pour un ou plusieurs ménages" (art.1), définition qui dépasse le sens étroit donné à "travaux ménagers" en incluant tous les pans du travail reproductif

\footnotetext{
18. Pour une discussion plus détaillée sur la politique d'État, voir Tomoko Nakamatsu, op. cit. 19. "Nenkinhokenryo zero no tsuma, otto ga ko shunyu hodo oku yushikisha chosa" ("Épouse à cotisation retraite zéro, mari à revenu très élevé, enquête d'expert”), in Asahi Shinbun (quotidien), 4 février 2012. Les résultats de l'enquête montrent que "73\% des épouses dont le mari gagne plus de 9 millions de yens ont un contrat d'assurance retraite de catégorie 3 [personnes qui ne cotisent pas directement mais sont couvertes par les cotisations de leur conjoint], la proportion est seulement de 32 \% des épouses si le mari gagne moins de 3 millions de yens par an." 20. Ruri Ito, "Immigration et travail de care dans une société vieillissante : le cas du Japon", in Jules Falquet, et al. (dir.), Le Sexe de la mondialisation : Genre, classe, race et nouvelle division du travail, Paris, Presses de Sciences Po, pp. 137-150. 21. Elles sont embauchées par différents types d'agences pour travailler soit dans des établissements de soins de longue durée, soit dans les centres d'accueil de jour, soit encore chez les particuliers. Dans le recensement, les home helpers sont dans la catégorie des personnels des services pour le soutien à la vie familiale. 22. Sachi Takahata, "Zainichi Firipinjin kaigosha, hito ashi saki yattekita 'Gaikokujin kaigo rōdōsha”" ("Les soignants philippins au Japon, pionnier du statut de 'travailleur soignant étranger"'), in Gendai shiso, vol. 37, $\mathrm{n}^{\circ}$ 2, 2009. 23. La Convention $\mathrm{n}^{\circ}$ 189 est assortie de la Recommandation $n^{\circ}$ 201. Pour le texte de la Convention $n^{\circ}$ 189, voir : http://www.ilo.org/ilolex/english/convdisp1.htm. Pour le texte de la Recommandation n²011, voir : http://www.ilo.org/ilolex/english/recdisp1.htm À présent, seuls 7 pays l'ont ratifiée : Uruguay, Philippines, Paraguay, Nicaragua, Maurice, Italie et Bolivie. Lors du vote pour l'adoption de la Convention à I'OIT, les représentants du gouvernement japonais et les délégués des travailleurs ont voté en faveur de la Recommandation et de la Convention, mais les délégués du patronat se sont abstenus pour la Convention et ont voté contre la Recommandation. Le journal Keidanren Times ${ }^{\circ} 3047$ du 30 juin 2011 a signalé que le Keidanren (Fédération des organisations économiques japonaises, c'est-à-dire l'organisation patronale des entreprises du Japon) s'est abstenu, jugeant impossible de ratifier la Convention, sans préciser les raisons. Cette organisation critique, par exemple, que les "heures de présence responsable" soient comptées comme heures à rémunérer (article 10-3 de la Convention 189).
} 
tels les soins aux personnes dépendantes ou aux enfants en bas âge. Ainsi, la catégorie des home helpers japonais peut y être incluse.

Ce qui est le plus important, c'est que la Convention prône la reconnaissance des travailleurs domestiques comme des "travailleurs" à part entière, c'est-à-dire garantit leur liberté d'association et de négociation collective, clarifie le contenu des contrats et des conventions de travail dans lesquels doivent être précisés le nombre d'heures de travail, les congés hebdomadaires, les congés payés, etc. En outre, la Convention déclare que ces points s'appliquent également aux travailleurs domestiques migrants (art. 8 et 15).

Comme le montre le tableau 1 , à l'exception de Hong Kong, nombreux sont les pays d'Asie où les travailleurs domestiques ne bénéficient pas du droit du travail national. Beaucoup de ces pays ne reconnaissent pas les droits élémentaires tels que le droit à un jour de congé par semaine, les heures de repos, les congés payés, le salaire minimal ou le congé maternitée ${ }^{24}$. L'adoption de la Convention $n^{\circ} 189$ établit un standard dont l'effet peut se voir déjà dans l'instauration d'un jour de congé hebdomadaire par le gouvernement de Singapour en janvier 2013. D'autres réformes sont en cours, aux Philippines, par exemple.

En ce qui concerne le Japon, les "employés de maison" sont exclus du code du travail promulgué et appliqué en $1947^{25}$. Ils ne bénéficient pas de l'application de la loi sur l'assurance chômage, ni de celle sur l'assurance couvrant les accidents du travail. Selon Hiromi Sakai et Ai Tamaki, cette exclusion était basée sur l'hypothèse que chaque ménage employant des "employés de maison" faisait preuve de prévenance à leur égard et que les employés bénéficiaient des "bonnes grâces" de leurs employeurs ${ }^{26}$. Mais plus de soixante ans sont passés depuis. Aujourd'hui, il existe donc la catégorie home helpers, avec une certaine dimension professionnelle réglementée par la loi sur l'assurance des soins de longue durée.

Or, paradoxalement, c'est la catégorie "employés de maison" qui sera appliquée aux travailleurs domestiques migrants accompagnant les "étrangers hautement qualifiés".

L'adoption de la Convention n 189 a été rendue possiblegrâce aux actions collectives menées par des réseaux transnationaux de travailleur(se)s domestiques, tels que l'International Domestic Workers' Network. À l'image des travailleurs domestiques migrants, les revendications des mouvements des travailleur(se)s domestiques ont traversé les frontières pour négocier un standard international. Quant au Japon, sa position apparemment "périphérique" dans la division internationale du travail reproductif ne peut quêtre illusoire, car beaucoup de Japonais en réalité emploient des travailleur(se)s domestiques à l'étranger.

La vie quotidienne de ces employés et de leur famille dépend du travail reproductif réalisé pour des employeurs japonais dans des pays en voie de développement. C'est ainsi que, contrairement à ce que les dirigeants japonais préconisent, le Japon n'est pas extérieur à la division internationale du travail reproductif. 